\title{
Habilidades dos residentes de pediatria na abordagem dos transtornos mentais na infância
}

\section{Pediatric residents' skills in addressing mental disorders in children}

Mônica Maria de Almeida Vasconcelos ${ }^{1}$, Lina Noor Constantin e Coutinho Vargas ${ }^{1}$, Aline Ferreira dos Santos ${ }^{1}$, Fabiana Maria Kakehasi $^{1}$

'Universidade Federal de Minas Gerais

\section{Palavras-chave:}

Internato e residência em pediatria; Pediatria; Educação médica; Transtornos mentais.

\section{Resumo}

Introdução: os transtornos mentais estão cada vez mais comuns no dia a dia do pediatra, sendo considerados 'distúrbios da modernidade'. Objetivo: avaliar a satisfação dos médicos-residentes com suas habilidades na abordagem de transtornos mentais na infância e sua percepção sobre o papel do pediatra no diagnóstico e abordagem desses distúrbios. Métodos: estudo envolvendo médicos-residentes de um programa de residência médica em pediatria, entre agosto de 2015 e maio de 2016. Utilizou-se questionário semiestruturado com informações sobre experiência e interesse dos participantes em diagnosticar transtornos mentais na rotina de atendimento da residência em pediatria. Resultados: o questionário foi respondido por 51 residentes (82,3\%) do total de 62 médicos (distribuídos entre residentes do primeiro ano - 20; do segundo ano - 20; e do ano opcional 22 residentes). Os médicos relataram média de 20 atendimentos por semana em atividades da residência e estimavam que, em metade deles, constataram-se transtornos mentais, sendo os mais comuns o distúrbio de aprendizagem (20\%), seguido por depressão, ansiedade e transtorno de deficit de atenção com hiperatividade (10\% cada). 0 treinamento, durante a residência, para abordagem desses distúrbios foi considerado insuficiente entre 58,8\% dos entrevistados. A insatisfação quanto à própria abordagem de transtornos mentais foi relatada por 76,5\% dos entrevistados. Conclusões: constatou-se que os transtornos mentais são diagnósticos comuns na prática pediátrica, segundo a percepção clínica dos médicos, e verificou-se defasagem no treinamento dos médicos-residentes para diagnosticar e abordar esses problemas.

\section{Keywords:}

Pediatrics; Internship and residency; Education, medical; Mental disorders

\begin{abstract}
Introduction: mental disorders are becoming more frequent in pediatricians' daily work, being, this way, considered 'modernity disorders'. Objective: to evaluate the satisfaction of pediatric residents with their abilities in dealing with mental disorders and their perception of the pediatrician's role in diagnosing and treating those disorders. Methods: a survey study with residents of a pediatric residency program, during August/2015 until May/2016. A semi-structured questionnaire was applied, with information about their experience and interest in mental disorders diagnosis in the residency routine appointments. Results: the survey was filled by 51 residents (82.3\%) among 62 doctors (scattered among $201^{\text {st }}$ residents, $202^{\text {nd }}$ year residents and 22 optional year residents). Doctors reported 20 appointments a week on average and estimated that mental disorders occur in half of them, the most common being learning disorders (20\%), depression, anxiety and attention deficit disorder with hyperactivity (10\% each). Training during residency to approach these problems was considered insufficient for $58.8 \%$ of them. Dissatisfaction related to their own approach to mental disorders was reported on $76.5 \%$ of surveyed. Conclusions: it was found that mental disorders are very common in pediatric practice according to doctors' clinical perception and a gap was verified in resident training to approach those problems.
\end{abstract}




\section{INTRODUÇÃO}

Os transtornos mentais afetam de forma significativa o bem-estar das crianças a tal ponto que hoje são chamados de 'distúrbios da modernidade'. Vários estudos evidenciam que até $20 \%$ das crianças e adolescentes apresentam ou apresentarão esses distúrbios. ${ }^{1,2,3,4}$ É crescente a preocupação mundial com a abordagem desses aspectos no dia a dia do pediatra, ${ }^{1}$ pois já foi evidenciado que cerca de $63,7 \%$ desses diagnósticos são feitos durante consultas de rotina. ${ }^{5}$ Identificar e conduzir os transtornos mentais deve fazer parte da atuação do pediatra na atenção primária. Apesar disso, existe uma enorme lacuna no treinamento dos pediatras durante a residência médica nas áreas da saúde mental, 2,4,6,7,8,9,10 associada à pouca disponibilidade na prática clínica para o atendimento desses casos.

Associa-se a este cenário o grande avanço no entendimento do desenvolvimento do cérebro e do comportamento nas últimas décadas, que não foi acompanhado por revisões na estrutura acadêmica tradicional. ${ }^{2,3} \mathrm{O}$ isolamento da atuação de algumas áreas e a falta de oportunidades interdisciplinares reais são barreiras persistentes na medicina acadêmica. Para melhorar a prática clínica, treinamento das novas gerações e pesquisa científica serão necessários para que os centros acadêmicos adotem passos importantes para desafiar as fronteiras das diversas áreas. Além de desejáveis, as mudanças são imperativas para alcançar uma abordagem realmente inovadora e mais eficiente no tratamento de transtornos mentais da criança na pediatria moderna. ${ }^{11}$

O objetivo deste estudo foi avaliar a satisfação dos médicos-residentes com as suas habilidades em lidar com transtornos mentais na infância e descrever sua percepção sobre o papel do pediatra no diagnóstico e abordagem desses distúrbios.

\section{MÉTODO}

Foram entrevistados médicos-residentes de um programa de residência em pediatria de uma instituição pública dos anos de 2014 e 2015. Trata-se de um hospital universitário, de atendimento terciário, que inclui em sua clientela muitos pacientes portadores de doenças crônicas e complexas. O questionário foi apresentado a 62 médicos (distribuídos entre residentes do primeiro ano - 20; do segundo ano - 20; e de especialidade pediátrica - 22 residentes). Ele foi desenvolvido pelos pesquisadores, abordando três aspectos:

a) prática médica;

b) interesse na capacitação e satisfação na abordagem de transtornos mentais; c) responsabilidade na identificação e abordagem desses distúrbios.

Este instrumento incluía também informações sobre idade, sexo, ano e instituição de graduação.

As respostas eram apresentadas, em uma escala ordinal simétrica, com categorias crescentes para as perguntas sobre treinamento adquirido, interesse e satisfação com a habilidade clínica de lidar com os transtornos mentais. Sobre a percepção do papel do pediatra na abordagem desses problemas, as respostas se apresentavam em três itens: concordo totalmente; concordo parcialmente; e não concordo. Ainda incluíam perguntas abertas sobre a experiência dos participantes com problemas específicos da saúde mental em pediatria, como transtorno de deficit de atenção e hiperatividade (TDAH), depressão, ansiedade e transtornos de aprendizagem.

As análises foram realizadas utilizando o SPSS versão 21 , iniciando com descritiva simples (com interquartil $25-75 \%$ ) e seguiu com a aplicação de testes de associação como qui-quadrado (proporções), teste T de Student e teste de Wilcoxon (médias e medianas). Foram utilizadas razões de chance (odds ratio - OR) com intervalo de confiança (IC) de $95 \%$ para quantificar a associação entre os fatores. A análise dos dados obtidos foi feita em tabelas de $2 \times 2$, com as respostas agrupadas nas categorias satisfação (satisfeito e muito satisfeito) e insatisfação (muito insatisfeito e algo insatisfeito), ou categorias de concordância (concordo totalmente/parcialmente e não concordo) e separadas de acordo com residentes de pediatria geral e de especialidades. Nas situações em que não foi possível utilizar o teste qui-quadrado, aplicou-se o teste exato de Fisher.

Este estudo foi aprovado pelo Comitê de Ética em Pesquisa da Universidade Federal de Minas Gerais (CAAE: 46519915.6.0000.514), e todos os participantes assinaram o Termo de Consentimento Livre e Esclarecido para participação no estudo, de acordo com a Resolução do Conselho Nacional de Saúde n. 466, de 2012.

\section{RESULTADOS}

Abaixo estão relatadas as informações contidas no questionário respondido pelos residentes.

\section{CARACTERÍSTICAS DOS PARTICIPANTES}

Durante os meses de agosto de 2015 a maio de 2016, foram entrevistados 51 residentes de pediatria de um universo de 62 médicos - 82,3\% (distribuídos entre residentes do primeiro ano - 20; segundo ano - 20; e opcional - 22 residentes), com mediana de idade 27 anos 
(IQ 25-75\%: 26-29), a maioria do sexo feminino (78,4\%; $40 / 51)$, a maior parte graduada em faculdades públicas federais $(74,5 \%)$, do programa de residência em pediatria geral $(70,5 \%)$ e em especialidades pediátricas $(29,5 \%)$. De acordo com o ano de formatura, verificou-se que $72,6 \%$ dos residentes formaram-se há menos de cinco anos.

Os médicos-residentes relataram média de 20 atendimentos (mín. 5 - máx. 70) por semana em atividades da residência e estimavam que em metade destes transtornos mentais foram observados. Dificuldade escolar foi relatada em $20 \%$ dos casos, seguida de sintomas de ansiedade e/ou tristeza intensa (depressão e ansiedade) em $10 \%$ e hiperatividade e/ou desatenção (TDAH) também em $10 \%$ dos casos. É importante ressaltar que esses diagnósticos eram presumidos por meio de observações clínicas dos residentes participantes ou de informações contidas em prontuário, e não através da utilização de instrumentos específicos para a realização desses diagnósticos.

\section{INTERESSE NA DISCUSSÃO DOS TRANSTORNOS MENTAIS}

Ambos os grupos de residentes de pediatria geral mostraram-se algo ou muito interessados na discussão do tema de transtornos mentais. Entretanto, notou-se diminuição significativa do interesse entre os residentes que cursam pediatria geral quando comparados com os da área de atuação (de 91,8\% para 50\%, respectivamente $\mathrm{p}=0,002$ ) (Tabela 1 ).

\section{TREINAMENTO NO PROGRAMA DE RESIDÊNCIA EM TRANSTORNOS MENTAIS}

O treinamento durante a residência para abordagem de transtornos mentais foi relatado como pouco suficiente ou insuficiente por mais da metade $(58,8 \%)$ dos médicos-residentes em ambos os grupos (Tabela 1).

\section{SATISFAÇÃO COM AS HABILIDADES EM ABORDAR TRANSTORNOS MENTAIS}

A descrição geral da habilidade em utilizar as técnicas de abordagem está descrita na figura 1 . Exceto na técnica de entrevista $(52,9 \%$ satisfeitos e $11,8 \%$ muito satisfeitos), observou-se insatisfação em mais de $70 \%$ dos médicos-residentes em todas as habilidades de abordagem dos transtornos mentais: realização de diagnóstico segundo os critérios da quinta edição do Manual de Diagnóstico e Estatística dos Transtornos Mentais (DSM-V) - 73,4\%; estratégias de aconselhamento - 81,3\%; manejo de dose de medicamentos antidepressivos $-83,7 \%$; aplicação de questionários de triagem diagnóstica - 93,8\%; e manejo de medicamentos utilizados no tratamento do TDAH $-95,9 \%$.

Na análise comparativa entre os grupos de residentes de pediatria e de área de atuação (Tabela 2), não houve diferença estatisticamente significativa entre as duas categorias $(p>0,05)$ quanto à satisfação com as técnicas de abordagem.

\section{RESPONSABILIZAÇÃO DO TRATAMENTO}

Houve divergência entre os residentes de pediatria geral e de área de atuação na responsabilização do manejo de pacientes com transtornos mentais. Os residentes de área de atuação acreditam, em sua maioria $(91,6 \%)$, que devem ser capazes de identificar esses transtornos e fazer o encaminhamento ao profissional de saúde mental. Os residentes de pediatria geral acham que são capazes de abordar esses casos (15/36; 41,7\%). Mesmo neste grupo, houve divergência na necessidade de responsabilização e encaminhamento.

\section{DISCUSSÃO}

Verificou-se a alta prevalência de transtornos mentais nos diagnósticos da população atendida pelos médicos-residentes, salientando que, como não houve treinamento específico dos residentes para realização de diagnósticos psiquiátricos, os transtornos listados foram provenientes de impressões clínicas dos entrevistados. Esses achados são concordantes com estudos previamente publicados, nos quais os transtornos mentais apresentaram prevalências de 10 a $50 \%$ na população pediátrica. ${ }^{12,13,14}$

Diante da população atendida pelo hospital de referência, um centro terciário, responsável pelo atendimento de casos de alta complexidade, esperava-se que esses diagnósticos fossem prevalentes como o estudo demonstrou. Entretanto, o estudo não conseguiu definir o cenário exato no qual os diagnósticos foram aventados, se na atenção primária ou em outros cenários de maior complexidade de assistência. Verificou-se que não havia programa específico de discussão desses temas no calendário de atividades teóricas dos médicos-residentes, o que poderia ter contribuído para sensação de insuficiência. A discussão do tema deve ser clara e pontuada já no decorrer do primeiro ano de residência, visto a importância do entendimento do conceito, num momento relevante para formação e aquisição de habilidades na abordagem dos pacientes. ${ }^{15}$

Nas duas a três últimas décadas, as demandas dos problemas de saúde das crianças e suas famílias têm sofrido 
Tabela 1 - Percentual do grau de interesse em transtornos mentais e percepção de médicos-residentes sobre treinamento na abordagem desses transtornos da infância

\begin{tabular}{|c|c|c|c|}
\hline Características & $\begin{array}{l}\text { Residentes de pediatria geral } \\
\text { n (\%) }\end{array}$ & $\begin{array}{c}\text { Residentes de especialidades } \\
\text { pediátricas } \\
\mathbf{n}(\%)\end{array}$ & Estatística $^{a}$ \\
\hline \multicolumn{4}{|l|}{ Interesse } \\
\hline Neutro & $3(8,1)$ & $7(50,0)$ & \\
\hline Algo/muito interessado & $34(91,8)$ & $7(50,0)$ & 0,002 \\
\hline \multicolumn{4}{|l|}{ Treinamento } \\
\hline Insuficiente/pouco & $21(56,8)$ & $9(64,3)$ & \\
\hline Suficiente (satisfatório + bom) & $16(43,2)$ & $5(35,7)$ & 0,754 \\
\hline
\end{tabular}

aTeste de Fisher.

Tabela 2 - Análise comparativa da insatisfação entre os residentes de pediatria geral e de áreas de atuação na habilidade em utilizar as técnicas de abordagem de transtornos mentais em crianças

\begin{tabular}{lccc}
\hline Habilidade & $\begin{array}{c}\text { Residentes de pediatria geral } \\
\mathbf{n}(\%)\end{array}$ & $\begin{array}{c}\text { Residentes de área de atuação } \\
\mathbf{n}(\%)\end{array}$ & p (Fisher) \\
\hline Técnicas de entrevista & $14(28,6)$ & $3(6,1)$ & 0,33 \\
Diagnóstico pelo DSM-V & $28(57,1)$ & $8(18,4)$ & 0,40 \\
Manejo de doses antidepressivos & $33(67,3)$ & $8(16,3)$ & 0,09 \\
Manejo de doses medicamentos TDAH & $36(73,5)$ & $11(22,4)$ & 0,43 \\
Estratégias de aconselhamento & $30(62,5)$ & $9(18,8)$ & 0,40 \\
Aplicação de questionários de triagem & $33(68,8)$ & $12(25)$ & 0,41 \\
transtornos mentais & & \\
\hline
\end{tabular}

Figura 1 - Percentual do grau de satisfação dos residentes com as técnicas de abordagem de transtornos mentais na infância




uma transformação significativa, com diminuição das doenças infecciosas graves e crescente reconhecimento de crianças com doenças crônicas e transtornos mentais. Isso direciona para que os programas de residência em pediatria se adequem para melhor treinamento na área de pediatria comportamental.

O Programa de Residência em Pediatria Geral, no qual foi realizado este estudo, já aderiu à extensão do treinamento em serviço com a inclusão de um terceiro ano, em que há oferecimento de estágios com ênfase em saúde mental, além de buscar melhor capacitação dos seus preceptores sobre temas relacionados. Espera-se que o presente trabalho potencialize melhor abordagem de temas relacionados à saúde mental na criança, por permitir aos residentes a conscientização de insuficiência em relação ao tema, dessa forma buscando aprofundamento nesses aspectos.

O interesse dos residentes em transtornos mentais na infância foi observado na grande maioria $(92 \%)$ dos residentes da pediatria geral. Devido ao aumento, na prática pediátrica diária, da prevalência dos problemas de saúde mental e dos fatores de risco psicossociais associados, era de se esperar que houvesse crescente interesse na abordagem do modelo biopsicossocial em vez do modelo biológico. Entretanto, viu-se que no decorrer dos anos de formação e, em especial, nas áreas de atuação, o tema 'transtorno mental' desperta menor interesse nos médicos-residentes, o que pode ser apreendido pela queda para $50 \%$, quando comparado com os residentes da pediatria geral que estavam nos primeiros anos de treinamento. ${ }^{16}$ Mesmo com o menor número de entrevistas, a redução do interesse justifica-se pela menor valorização histórica da abordagem de saúde mental, associada à maior exposição a doenças crônicas na subárea de atuação. Insere-se, assim, carga de estudo adicional ao residente.

Notou-se que os residentes se apresentaram satisfeitos apenas com as técnicas de entrevista, e que a insatisfação predominava em todos os demais aspectos avaliados (diagnóstico, estratégias de aconselhamento, manejo de doses dos medicamentos e aplicação de questionários de triagem para os principais transtornos psiquiátricos), inclusive entre os residentes de área de atuação. Pode-se inferir que, apesar da exposição a essas situações, os médicos-residentes deparam-se sem treinamento e desestimulados a buscar ativamente identificação e abordagem desses distúrbios. ${ }^{17}$ Apesar de pediatras há mais tempo e, teoricamente, ter tido mais contato com casos de depressão, TDAH e ansiedade, os residentes das áreas de atuação mostraram-se insatisfeitos até mesmo com suas estratégias de aconselhamento e aplicação de questionários de triagem para os transtornos mentais, confirmando a defasagem no treinamento durante a residência. ${ }^{18,19}$

Não houve consenso entre os dois grupos de residentes sobre a percepção da responsabilidade de abordar os pacientes com transtornos mentais. A maioria dos residentes da pediatria geral reconheceu que são eles os responsáveis em diagnosticar e tratar esses pacientes, enquanto os da área de atuação acreditaram que deveriam encaminhá-los para a abordagem pelos profissionais da saúde mental. Essa indecisão sobre a responsabilidade da abordagem dos distúrbios foi descrita em outros estudos, ${ }^{20,21}$ nos quais não está amplamente aceita essa responsabilidade de tratar por parte dos pediatras que atuam na atenção primária.

A abordagem do médico-pediatra aos transtornos mentais também pode ser variável, de acordo com o local em que atuam, o tempo após o término do treinamento e a percepção do impacto dessas condições na saúde de seus pacientes. ${ }^{22,23}$ A diferença que se observou entre os dois grupos estudados justifica-se pelo autorreconhecimento dos residentes da área de atuação como profissionais que atuariam na atenção secundária ou terciária e não mais se deparando com esses transtornos mentais na fase de diagnóstico inicial, como ocorre na atenção primária.

O presente trabalho apresentou como ponto forte a alta proporção de respondentes, com respostas anônimas, além de garantir o aspecto de voluntariedade de escolha em participar ou não da pesquisa, condições indispensáveis para redução de vieses. Entretanto, observam-se limitações, como o estabelecimento de questões em formato de perguntas fixas e abrangentes, que podem não ter facilitado o diagnóstico situacional mais preciso. Além disso, algumas situações clínicas podem ter sido vivenciadas há um intervalo de tempo longo em relação à participação nesta pesquisa, o que contribuiria ao viés memória. Apesar do número pequeno de entrevistados, por se tratar de uma amostra de um único serviço de residência pediátrica, trata-se de estudo inédito no nosso meio.

\section{CONCLUSÕES}

Verificou-se uma lacuna no treinamento dos médicos-residentes para a abordagem dos transtornos mentais, apesar do interesse desses profissionais sobre o tema. Há também uma insatisfação dos residentes com sua própria habilidade em lidar com esses problemas.

Esperamos que os resultados apresentados neste estudo possam auxiliar os coordenadores dos programas de residência médica a adequar cada vez mais as 
experiências de seus residentes, aperfeiçoando o treinamento na abordagem de forma que eles se sintam mais satisfeitos e seguros no diagnóstico e no manejo dos transtornos mentais na infância.

\section{AGRADECIMENTOS}

Agradecemos o apoio da Coordenação de Aperfeiçoamento de Pessoal de Nível Superior (Capes), da qual Mônica Maria de Almeida Vasconcelos foi bolsista de agosto de 2016 a junho de 2017 (processo n. 2769/15-8)

Atlanta Developmental Disabilities Study. Pediatrics. 1992;89(4 Pt 1):624-30.

13 Boyle CA, Decoufle P, Yeargin-Allsopp M. Prevalence and health impact of developmental disabilities in US children. Pediatrics. 1994;93(3):399-403.

14 Lavigne JV, Binns HJ, Christoffel KK, Rosenbaum D, Arend $\mathrm{R}$, Smith K et al. Behavioural and emotional problems among pre-school children in pediatric primary care: prevalence and pediatricians' recognition. Pediatrics Practice Research Group. Pediatrics. 1993;91(3):649-55.

15 Phillips S, Friedman SB, Smith J, Felice ME. Evaluation of a residency training program in behavioral pediatrics. Pediatrics. 1983;71(3):406-12.

16 Committee on Psychosocial Aspects of Child and Family Health. American Academy of Pediatrics. The new morbidity revisited: a renewed commitment to the psychosocial aspects of pediatric care. Pediatrics. 2001;108(5):1227-30.

17 Lykke K, Graungaard AH, Ertmann R, Reventlow S. The Danish preventive child health examination should expand on mental health and the well-being of the family. Dan Med J. 2015;62(5):A5060.

18 Applegate H, Kelley ML, Applegate BW, Jayasinghe IK, Venters CL. Clinical case study: pediatric residents' discussions of and interventions for children's behavioral and emotional problems. J Pediatr Psychol. 2003;28(5):315-21.

19 Brown R, Doonan S, Shellenberger S. Using children as simulated patients in communication training for residents and medical students: a pilot program. Acad Med. 2005;80(12):1114-20.

20 Boreman CD, Thomasgard MC, Fernandez SA, Coury DL. Resident training in developmental/behavioral pediatrics: where do we stand? Clin Pediatr (Phila). 2007;46(2):135-45.

21 Mullan PB, Wolf FM, Ertel IJ. Residents' evaluation of behavioural paediatrics instruction. Med Educ. $1989 ; 23(5): 447-52$.

22 Guevara JP, Rothbard A, Shera D, Zhao H, Forrest CB, Kelleher $\mathrm{K}$ et al. Correlates of behavioral care management strategies used by primary care pediatric providers. Ambul Pediatr. 2007;7(2):160-66.

23 Olson A, Kelleher K, Hammond C, Zuckerman B, Dietrich A. Primary care pediatricians' roles and perceived responsibilities in the identification and management of maternal depression. Pediatrics. 2002;110(6):1169-76. 